\title{
Standard and magnetically controlled growing rods for the treatment of early onset scoliosis
}

\author{
Ilkka J. Helenius
}

Department of Paediatric Orthopaedic Surgery, University of Turku and Turku University Hospital, Turku, Finland

Correspondence to: Ilkka J. Helenius, MD, PhD. Department of Paediatric Orthopaedic Surgery, University of Turku and Turku University Hospital, Kiinamyllynkatu 4-8, 20520 Turku, Finland. Email: ilkka.helenius@utu.fi.

\begin{abstract}
Distraction based spinal instrumentation represents the most common and standard surgical technique to correct early onset scoliosis (EOS), i.e., scoliosis which has been diagnosed before the age of 10 years. Surgical treatment of EOS aims at controlling spinal deformity while maintaining spinal growth which is mandatory for the development of normal lung capacity. To achieve these goals the spinal instrumentation needs to be distracted to facilitate spinal growth during treatment. Distraction can be obtained by repeated surgical lengthenings (traditional growing rods, TGRs) or using magnetically controlled growing rods (MCGRs), which can be lengthened using external remote controller on an outpatient basis. The outcomes of TGR instrumentation for EOS are well described with follow-up until skeletal maturity: normal spinal growth can be maintained, $40-50 \%$ of the scoliosis can be corrected, but there is an over $50 \%$ risk of complications including deep wound infection, rod failure, and instrumentation pull-out. MCGR instrumentation may reduce the risk of wound related complications, provides similar deformity correction, but may not provide as much spinal growth. Metallosis around the instrumentation necessitates MCGR removal and definitive final instrumented fusion at the end of growth friendly management. Even severe EOS can be treated using distraction based spinal instrumentation.
\end{abstract}

Keywords: Early onset scoliosis (EOS); traditional growing rods (TGRs); magnetically controlled growing rods (MCGRs); complications; spinal fusion

Submitted Aug 07, 2019. Accepted for publication Sep 11, 2019.

doi: 10.21037/atm.2019.09.72

View this article at: http://dx.doi.org/10.21037/atm.2019.09.72

\section{Introduction}

Early onset scoliosis (EOS) is defined as a scoliosis, which occurs before the age of 10 years (Figures 1,2$)(1,2)$. If EOS is left untreated or if treated by spinal fusion resulting into a short trunk and spinal height, EOS is associated with increased morbidity and even mortality (3-5). EOS is a heterogeneous disorder and therefore a widely accepted classification has been published (1). This classification includes age, etiology (congenital or structural, neuromuscular, syndromic and idiopathic), magnitude of scoliosis and kyphosis as well as rate of deformity progression. Growth-friendly management is indicated for progressive scoliosis $(4,6,7)$. EOS may progress rapidly during early growth and, thus, early diagnosis and referral to a pediatric spine unit is necessary $(1,7)$.

Spinal instrumentation is needed in the surgical treatment of EOS to correct scoliosis and to allow spinal growth, which is necessary for normal development of lung capacity (8-11). Skaggs et al. (12) have provided a classification for growth-friendly instrumentation used in the surgical management of EOS. They divided the type of instrumentation into distraction based (e.g., growing rods) (Figures 3-5), growth-guidance (e.g., Luque-Trolley or Shilla), and compression based (e.g., stapling or tethering).

This review will focus on the surgical treatment of EOS using distraction based spinal instrumentation either traditional growing rods (TGRs) or using magnetically controlled growing rods (MCGR). 


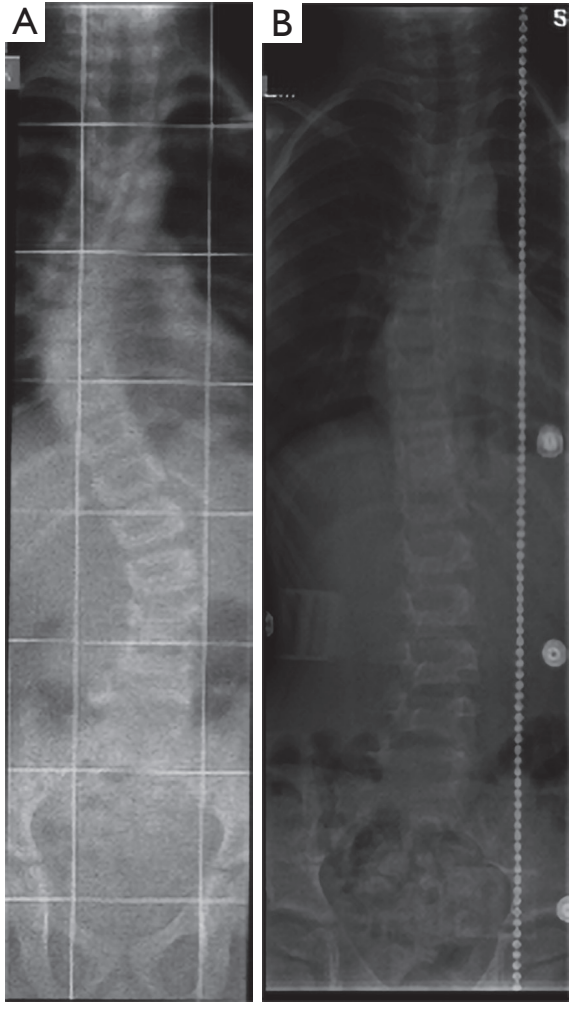

Figure 1 Two-year-old boy with syndromic 50-degree early onset scoliosis (Marfan syndrome). (A) Standing posteroanterior spinal radiographs before casting; (B) 1-year follow-up at the end of casting, radiograph taken in a brace.

\section{Indications for growing rod surgery}

EOS can be treated with casting, bracing or surgery $(4,7,13,14)$ (Figures $1-5)$. Serial casting is indicated for progressive infantile scoliosis (scoliosis diagnosed before the age of 3 years) (13) (Figure 1), while growth-friendly surgery is indicated when scoliosis is progressing to over $50^{\circ}$ and conservative management has failed $(4,6,7,14)$ (Figures 2-5). Progressive and non-progressive idiopathic infantile scoliosis are differentiated by using the rib-vertebra angle difference (RVAD) (15). A RVAD of $20^{\circ}$ or more is typical of progressive infantile scoliosis. A clear indicator for progressive infantile scoliosis is the apical rib head in phase two. In this stage, the rib head overlaps the corresponding apical vertebral body (15).

Growing rod surgery is associated with high risk of complications $(6,16,17)$ (Figures 4,5). Bess et al. (16) found a minimum one complication in $81(58 \%)$ out of 140 children treated using a growing rod instrumentation over a minimum 5-year follow-up. Older age and

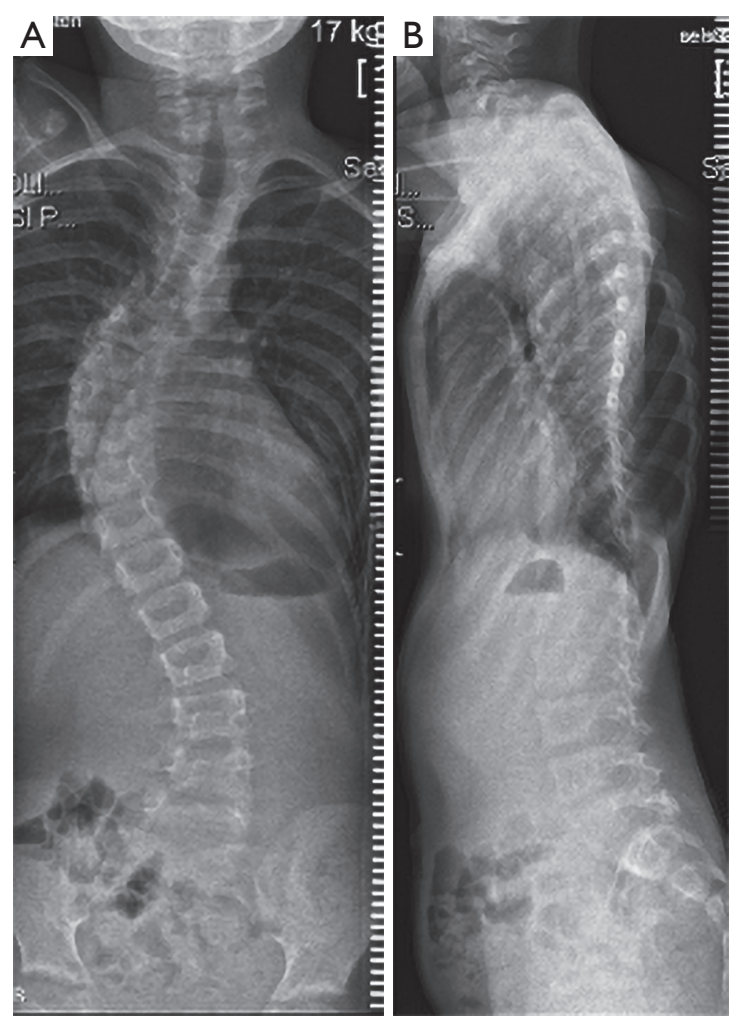

Figure 2 The same boy at the age of 5 years and 8 months before surgery. (A) Standing posteroanterior spinal radiograph; (B) lateral spinal radiograph. Radiographs show 65-degree thoracic scoliosis with hypokyphosis.

subfascial instrumentation decreased this risk, while every surgery increased the risk of complication by $24 \%$. Postponing growing rod surgery 1 year reduced the risk of complications by $12 \%$. Therefore, Fletcher et al. (18) have popularized delaying tactics in the surgical management of EOS. Spinal deformity can be controlled by using cast or brace, and in this manner surgery can be postponed several years and thus reducing risks of repeated surgery (Figure 1A,B). Further growth of spine results into larger bony structures, which allow bigger implants and therefore more solid spinal instrumentation. This reduces risks of implant related complications but also the need for repeated procedures. Halo-gravity traction can be used to facilitate larger curves to be amenable for casting under general anaesthesia (18). The upper limit for delaying tactics to be an EOS of 90 degrees or more $(19,20)$. A severe curve above 90 degrees at the time on initial management results into large residual curve and increases significantly the risks of complications (19). 


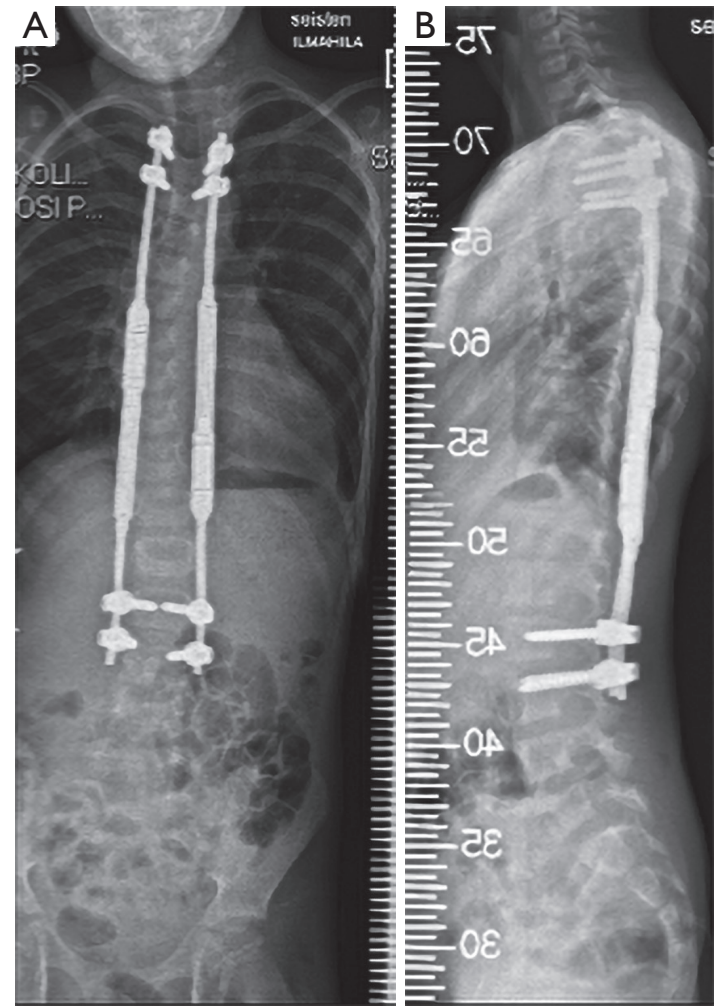

Figure 3 Standing posteroanterior (A) and lateral (B) spinal radiograph after surgery using magnetically controlled growing rods. Fixation points using pedicle screws.

\section{Surgical technique and outcomes}

Growth-friendly spinal instrumentation options for EOS include growing rods (TGR or MCGR) (4,6,7,21-24), Shilla and other growth guidance systems (25), and a vertical expandable prosthetic titanium rib (VEPTR) $(26,27)$. At skeletal maturity segmental instrumentation and final spinal fusion can be performed $(28,29)$.

Growing rod instrumentation has been the main surgical method to address EOS and several studies have documented follow-up until skeletal maturity with final fusion or observation only after last surgical lengthening $(4,6,7,16,17,20,24,28,29)$. TGR is a growth-friendly surgical management, which requires repeated lengthenings but is associated with a high risk of complications $(16,17,20)$. These include deep surgical site infection, rod fractures and failure of proximal fixation (Figure 4A,B). MCGRs are a new distraction-based spinal instrumentation, which allows outpatient construct lengthenings (21-24). This may reduce the risk of deep wound infection (Figure 3A,B) (21-24). VEPTR instrumentation is typically reserved for patient
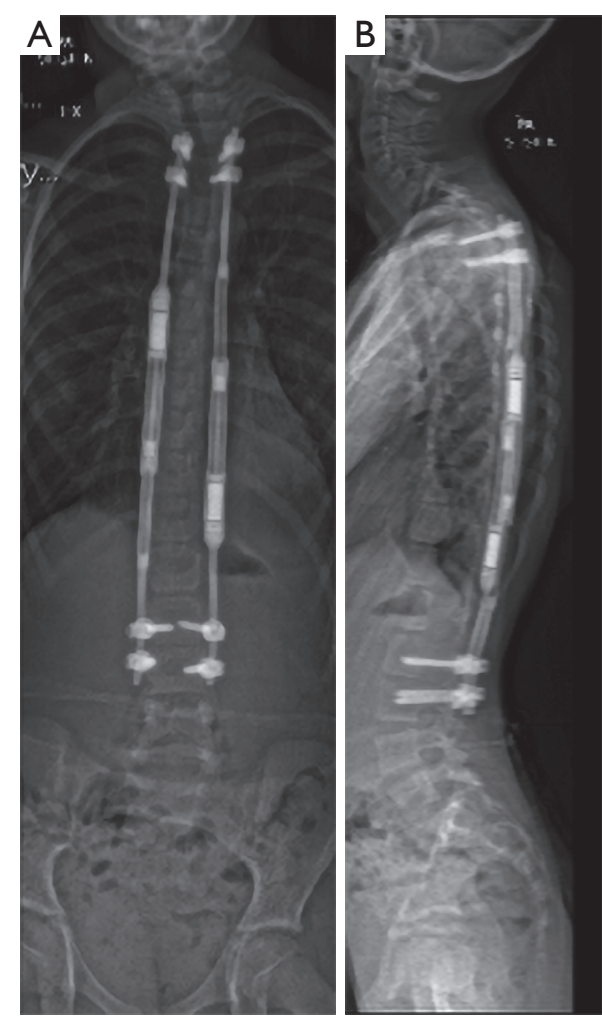

Figure 4 Standing posteroanterior (A) and lateral (B) spinal radiograph 3 years after index surgery at the age of 8 years. Radiographs demonstrates well-aligned and corrected spinal deformity, but the proximal thoracic pedicle screws seem to be pulling out.

with fused ribs $(14,26,27)$.

Both TGRs and MCGRs are used in a sub-muscular, dual-rod fashion (4,7,21-24). Fixation points are exposed using two small separate incisions and the rest of the spine is left untouched (4). Typical construct involves upper thoracic pedicle screws or laminar hooks and pedicle screws in the mid-lumbar spine (Figure 3A,B) (7). Rods are tunneled from the upper incision submuscularly to the lower incision. Growing rods provide correction of the spinal deformity using indirect methods: distraction on the concave side and cantilevering on the convexity of the curve resulting into roughly $40 \%$ correction of scoliosis $(4,6,7)$. Severe EOS (defined as major curve $>90^{\circ}$ ) remains difficult to correct using growing rods $(19,20)$. Coronal curve correction remains lower ( $43 \%$ in this study) using growing rods than when using high-density pedicle screw instrumentation in children undergoing surgery for adolescent idiopathic scoliosis $(>60 \%)$. Therefore, a significant residual curve 

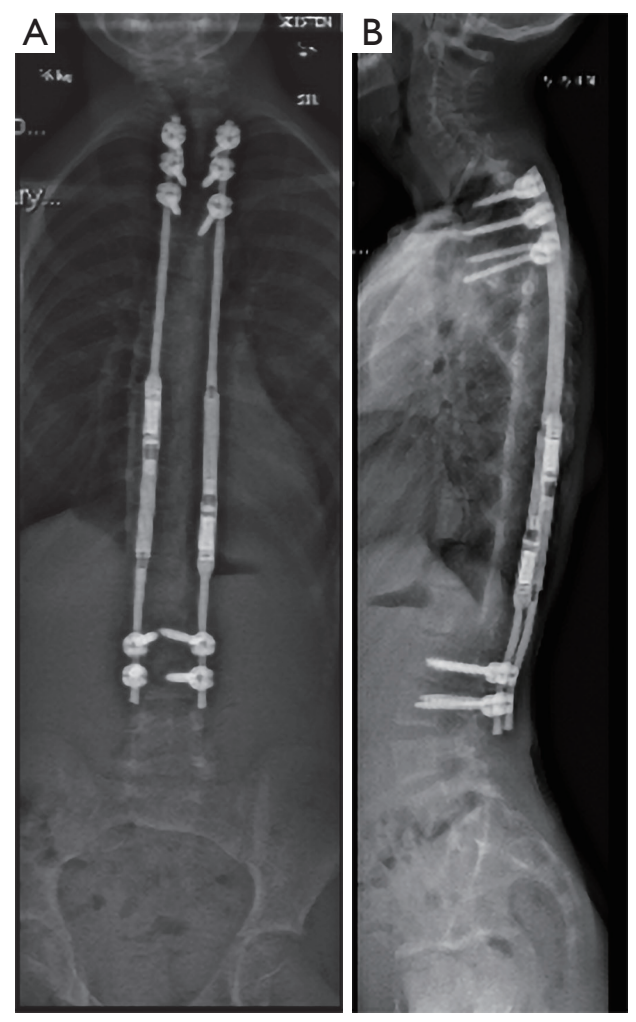

Figure 5 Standing posteroanterior (A) and lateral (B) spinal radiograph at the age of 9 years. Radiographs 6 months after revision surgery, where all fixation points were revised. This boy had a transient intraoperative neuromonitoring change (loss of left sided motor evoked potentials), while revising the proximal anchors. Postoperatively he was neurologically fully intact.

remains at the end of growth-friendly management $(4,6,7,30)$.

In a typical surgical setting a standard MCGR rod (distraction towards caudal direction) is applied on the left side and an MCGR offset rod (distraction towards cranial direction) is applied on the right side (Figures 3,4) (21-24,31). This construct allows separate lengthening of the rods and minor adjustment of the coronal balance. By placing two standard MCGR rods may allow both rods lengthening at the same time and thus theoretically providing higher lengthening forces (31).

The initial growing rod surgery provides approximately $50 \%$ of the spinal length increase and the lengthenings provide the remaining half in children $(4,6,7)$. In a multicenter investigation, Akbarnia et al. (4) followed 23 EOS children (7 with idiopathic, 3 congenital, 13 secondary scoliosis) who were operated using TGRs with a minimum 2-year follow-up. All patients underwent lengthenings every 6 months. The average number of lengthenings per patient was 6.6 and this resulted into T1-S1 growth of 4.6 or $1.2 \mathrm{~cm} /$ year. Patients with congenital scoliosis received significantly less spinal length during the index surgery while lengthenings produced similar spinal growth. Distraction of the spine with instrumentation may stimulate growth of the spine, since annual growth of $1.2 \mathrm{~cm} /$ year exceeds that of the normal spine. The Growing Spine Study Group (31) has evaluated the spinal height gain over repeated surgical lengthenings (32). A decrease of T1$\mathrm{S} 1$ gain from $10 \mathrm{~mm}$ at first lengthening to $6 \mathrm{~mm}$ at 7 th lengthening occurred, but some gain occurred even after multiple lengthenings, i.e., phenomenon called "law of diminishing returns" (32).

The use of MCGRs is the latest technique allowing non-invasive lengthenings (Figure 4A,B) (21-24,31). Bilateral MCGRs are instrumented subfascial to the spine with pedicle screws or hooks to connect the proximal and the distal fixation of the rods. The MCGR contains a magnetically-driven lengthening mechanism. After the index surgery, lengthening can be done without anaesthesia with an external remote controller on an outpatient clinic. It has been suggested that, because there is no need for repeated surgeries, the risk of wound infections would be lower than with TGRs (21-23). MCGRs have been shown to be a safe and effective in children undergoing primary surgery for EOS $(21,22)$. However, patients who have had a TGR and are converted to MCGR seem to achieve less spinal growth (23). In one study, $47 \%$ of children operated using MCGR for EOS have required an unplanned reoperation during a minimum 2-year follow-up (21). The most common indications for re-operation were failure of distraction, proximal foundation failure, and rod breakage (Figure $4 A, B$ ). More frequent distractions (between 1 week and 2 months) were associated with a higher rate of reoperation than distraction frequencies between 3 and 6 months (21).

In severe EOS a preoperative halo traction is a useful additional corrective maneuver, since it has been shown to reduce thoracic kyphosis more effectively than anterior spinal discectomy in EOS, which reduces forces on the spinal instrumentation $(33,34)$. Preoperative halo traction has not been associated with severe complications in severe EOS (19).

Complications associated with growing rods include fixation point failure, rod fracture, autofusion, and increased risk of deep wound infection $(16,17)$. Growing 
rods for severe EOS have also been associated with a relatively high risk of neurological deficits (5\%) (19) The main mechanisms of the neurological deficits have been: (I) correction and distraction of the spine during initial surgery; (II) pedicle screw pull-out during followup; and (III) difficulties in placing thoracic pedicle screws during revision surgery (Figure $5 A, B)(19)$. To reduce the risk of upper thoracic pedicle screw related complications following recommendations have been suggested: in case of difficulties in obtaining a solid pedicle screw fixation at index surgery, consider using laminar or rib hooks; a minimum of two pairs of pedicle screws should always be used, although this may not fully prevent pull-out related neurologic complications; Revision of fixation points should be regarded as major surgery and the use of intraoperative monitoring is recommended (19).

\section{Comparison of traditional and MCGRs}

TGRs are lengthened surgically typically at 6-month interval (6). MCGRs can be lengthened more often, but more frequent distractions than 3 months appear to be associated with increased risk of instrumentation failure (21) and therefore most centers have elected to lengthen these devices at 3-month interval.

Noordeen et al. (35) have measured the forces needed to distract TGRs during surgery. Based on stiffening spine and surrounding soft tissues these forces increase during the treatment and after 6th lengthening forces up to 650 Newtons $(\mathrm{N})$ are required to provide the $10 \mathrm{~mm}$ lengthening of the instrumentation. MCGR provides a force of $230 \mathrm{~N}$ until the safety mechanism begins to stall or clicking $(23,36)$. The maximal force generated by MCGR decreases by $8 \%$ when the rod is lengthened up to $40 \mathrm{~mm}$ (36). Recently, explanted MCGR have been investigated (37). The duration the rods were in vivo was negatively correlated with the force produced on testing. After 38 months' use in vivo, explanted MCGR did not produce any force (37). This suggests these rods should be exchanged within 3 years after implantation (Figure $4 A, B$ ). The forces needed to keep up lengthening of spine may explain, why TGRs seem to provide more spinal growth than MCGR instrumentation does. This difference seems also clinically relevant in revision cases where TGRs should be given a priority (23). TGRs are also indicated in patients who need repeated MR images for medical followup, as MCGR prevents high quality MR images of the of spinal cord and perivertebral structures (38). Such follow- up might be needed, e.g., in the follow-up of children with neurofibromatosis.

TGR surgery with repeated surgical lengthenings results into autofusion and stiffening of the spine (39). The additional correction and spinal length obtained at final fusion has therefore remained very limited (28). Jain et al. (29) have reported that patients with acceptable spinal deformity and balance at the end of TGR management may just be followed up instead of final fusion. In contrast, patients with severe EOS at the beginning of growth friendly management may benefit from final fusion in terms of better spinal deformity correction and spinal length obtained (20).

Metallosis around the MCGR instrumentation has been reported in revision cases (40). This appears to be the result of micro-movement between the rod and the housing part of the device and consists of metal fragments of Titanium. As this metallosis appears to be clinically significant the MCGR instrumentation needs be removed at the end of growth friendly management and a definitive spinal instrumentation performed at the end of this management. Preliminary findings suggest that instrumented segments experience similar stiffness as with TGR thus limiting further correction and length gain during the final fusion (31).

\section{Conclusions}

Growing rods represent the standard and well documented surgical technique to address EOS even in its severe forms. TGRs allow reasonable correction of scoliosis and maintain adequate spinal growth, but are associated with relatively high risk of complications and unplanned re-operations due to repeated surgical lengthenings. MCGRs are currently widely used, but few patients have been followed up to skeletal maturity. Lengthenings on an outpatient basis represent a more advancement in the treatment of EOS, but more information is needed on the risk of metallosis during and other complications during treatment. Halogravity traction is a useful adjunct in severe EOS. TGR management results into stiffening and autofusion of the spine and therefore not all patients need final fusion, while patients with MCGRs should be treated by explantation of these rods and segmental spinal instrumentation and fusion at the end of growth friendly management.

\section{Acknowledgments}

The author thanks Medtronic, Innosurge As, and Turku 
University Hospital for supporting the writing of this manuscript.

Dr. Ilkka Helenius received grants from Industry and Institutions for preparation of this manuscript.

\section{Footnote}

Conflicts of Interest: Consultant for Medtronic; grants and research funding from Medtronic, K2M via Innosurge As.

Ethical Statment: The author is accountable for all aspects of the work in ensuring that questions related to the accuracy or integrity of any part of the work are appropriately investigated and resolved.

\section{References}

1. Williams BA, Matsumoto H, McCalla DJ, et al. Development and initial validation of the Classification of Early-Onset Scoliosis (C-EOS). J Bone Joint Surg Am 2014;96:1359-67.

2. El-Hawary R, Akbarnia BA. Early Onset Scoliosis - Time for Consensus. Spine Deform 2015;3:105-6.

3. Pehrsson K, Larsson S, Oden A, Nachemson A. Longterm follow-up of patients with untreated scoliosis. A study of mortality, causes of death, and symptoms. Spine (Phila Pa 1976) 1992;17:1091-6.

4. Akbarnia BA, Marks DS, Boachie-Adjei O, et al. Dual growing rod technique for the treatment of progressive early-onset scoliosis: a multicenter study. Spine (Phila $\mathrm{Pa}$ 1976) 2005;30:S46-57.

5. Karol LA, Johnston C, Mladenov K, et al. Pulmonary function following early thoracic fusion in nonneuromuscular scoliosis. J Bone Joint Surg Am 2008;90:1272-81.

6. Akbarnia BA, Breakwell LM, Marks DS, et al. Dual growing rod technique followed for three to eleven years until final fusion: the effect of frequency of lengthening. Spine (Phila Pa 1976) 2008;33:984-90.

7. Akbarnia BA. Management themes in early onset scoliosis. J Bone Joint Surg Am 2007;89:42-54.

8. Dimeglio A, Bonnel F. Le rachis en croissance (The spinal column in growth). Paris: Springer Verlag, 1990.

9. Dimeglio A. Growth of the spine before the age 5 years. J Pediatr Orthop B 1992;1:102-7.

10. Dimeglio A. Growth in pediatric orthopedics. In: Morrissy RT, Weinstein SL. editors. Lovell and Winter's pediatric orthopaedics. 6th edition. Philadelphia: Lippincott
Williams \& Wilkins, 2006:35-65.

11. Helenius IJ. Normal and abnormal growth of spine. In: Yazici M. editor. Non-idiopathic spine deformities in young children. Rolle: EFORT, 2011:3-13.

12. Skaggs DL, Akbarnia BA, Flynn JM, et al. A classification of growth friendly spine implants. J Pediatr Orthop 2014;34:260-74.

13. Mehta MH. Growth as a corrective force in the early treatment of progressive infantile scoliosis. J Bone Joint Surg Br 2005;87:1237-47.

14. Helenius IJ. Treatment strategies for early-onset scoliosis. EFORT Open Rev 2018;3:287-93.

15. Mehta $M H$. The rib-vertebra angle in the early diagnosis between resolving and progressive infantile scoliosis. J Bone Joint Surg Br 1972;54:230-43.

16. Bess S, Akbarnia BA, Thompson GH, et al. Complications of growing-rod treatment for early-onset scoliosis: analysis of one hundred and forty patients. J Bone Joint Surg Am 2010;92:2533-43.

17. Kabirian N, Akbarnia BA, Pawelek JB, et al. Deep surgical site infection following 2344 growing-rod procedures for early onset scoliosis. Risk factors and clinical consequences. J Bone Joint Surg Am 2014;96:e128.

18. Fletcher ND, McClung A, Rathjen KE, et al. Serial casting as a delay tactic in the treatment of moderate-to-severe early-onset scoliosis. J Pediatr Orthop 2012;32:664-71.

19. Helenius IJ, Oksanen HM, McClung A, et al. Outcomes of growing rod surgery for severe compared with moderate early-onset scoliosis: a matched comparative study. Bone Joint J 2018;100-B:772-9.

20. Helenius IJ, Sponseller PD, McClung A, et al. Surgical and Health-related Quality-of-Life Outcomes of Growing Rod "Graduates" With Severe versus Moderate Earlyonset Scoliosis. Spine (Phila Pa 1976) 2019;44:698-706.

21. Kwan KYH, Alanay A, Yazici M, et al. Unplanned reoperations in magnetically controlled growing rod surgery for early onset scoliosis with a minimum of twoyear follow-up. Spine (Phila Pa 1976) 2017;42:E1410-4.

22. Hosseini P, Pawelek J, Mundis GM, et al. Magnetically controlled growing rods for early-onset scoliosis: a multicenter study of 23 cases with minimum 2 years follow-up. Spine (Phila Pa 1976) 2016;41:1456-62.

23. Keskinen H, Helenius I, Nnadi C, et al. Preliminary comparison of primary and conversion surgery with magnetically controlled growing rods in children with early onset scoliosis. Eur Spine J 2016;25:3294-300.

24. Akbarnia BA, Pawelek JB, Cheung KM, et al. Traditional growing rods versus magnetically controlled growing rods 
for the surgical treatment of early-onset scoliosis: a casematched 2-year study. Spine Deform 2014;2:493-7.

25. McCarthy RE, McCullough FL. Shilla Growth Guidance for Early-Onset Scoliosis: Results After a Minimum of Five Years of Follow-up. J Bone Joint Surg Am 2015;97:1578-84.

26. Campbell RM Jr, Smith MD, Mayes TC, et al. The characteristics of thoracic insufficiency syndrome associated with fused ribs and congenital scoliosis. J Bone Joint Surg Am 2003;85:399-408.

27. Campbell RM Jr, Smith MD, Mayes TC, et al. The effect of opening wedge thoracostomy on thoracic insufficiency syndrome associated with fused ribs and congenital scoliosis. J Bone Joint Surg Am 2004;86:1659-74.

28. Flynn JM, Tomlinson LA, Pawelek J, et al. Growingrod graduates: lessons learned from ninety-nine patients who completed lengthening. J Bone Joint Surg Am 2013;95:1745-50.

29. Jain A, Sponseller PD, Flynn JM, et al. Avoidance of "final" surgical fusion after growing-rod treatment for early-onset scoliosis. J Bone Joint Surg Am 2016;98:1073-8.

30. Ledonio CG, Polly DW Jr, Vitale MG, et al. Pediatric pedicle screws: comparative effectiveness and safety: a systematic literature review from the Scoliosis Research Society and the Pediatric Orthopaedic Society of North America task force. J Bone Joint Surg Am 2011;93:1227-34.

31. Cheung JPY, Yiu K, Kwan K, et al. Mean 6-Year Followup of Magnetically Controlled Growing Rod Patients With Early Onset Scoliosis: A Glimpse of What Happens to Graduates. Neurosurgery 2019;84:1112-23.

32. Sankar WN, Skaggs DL, Yazici M, et al. Lengthening of dual growing rods and the law of diminishing returns. Spine (Phila Pa 1976) 2011;36:806-9.

Cite this article as: Helenius IJ. Standard and magnetically controlled growing rods for the treatment of early onset scoliosis. Ann Transl Med 2020;8(2):26. doi: 10.21037/ atm.2019.09.72
33. Caubet JF, Emans JB. Halo-gravity traction versus surgical release before implantation of expandable spinal devices: a comparison of results and complications in early-onset spinal deformity. J Spinal Disord Tech 2011;24:99-104.

34. Welborn MC, Krajbich JI, D'Amato C. Use of Magnetic Spinal Growth Rods (MCGR) With and Without Preoperative Halo-gravity Traction (HGT) for the Treatment of Severe Early-onset Scoliosis (EOS). J Pediatr Orthop 2019;39:e293-7.

35. Noordeen HM, Shah SA, Elsebaie HB, et al. In vivo distraction force and length measurements of growing rods: which factors influence the ability to lengthen? Spine (Phila Pa 1976) 2011;36:2299-303. Erratum in: Spine (Phila Pa 1976) 2012;37:432. Al Mukhtar, Mohannad [corrected to Al-Mukhtar, Mohannad].

36. Poon S, Spencer HT, Fayssoux RS, et al. Maximal Force Generated by Magnetically Controlled Growing Rods Decreases With Rod Lengthening. Spine Deform 2018;6:787-90.

37. Rushton PRP, Smith SL, Forbes L, et al. Force Testing of Explanted Magnetically Controlled Growing Rods. Spine (Phila Pa 1976) 2019;44:233-9.

38. Poon S, Chen YH, Wendolowski SF, et al. Cadaveric Study of the Safety and Device Functionality of Magnetically Controlled Growing Rods After Exposure to Magnetic Resonance Imaging. Spine Deform 2018;6:290-8.

39. Cahill PJ, Marvil S, Cuddihy L, et al. Autofusion in the immature spine treated with growing rods. Spine (Phila $\mathrm{Pa}$ 1976) 2010;35:E1199-203

40. Teoh KH, von Ruhland C, Evans SL, et al. Metallosis following implantation of magnetically controlled growing rods in the treatment of scoliosis: a case series. Bone Joint J 2016;98-B:1662-7. 